\title{
La India y la Alianza del Pacífico: oportunidades y desafíos*
}

\author{
Paola Andrea Baroni** y Hari Seshasayee**
}

\section{Resumen}

Históricamente, las vinculaciones políticas de la India con América Latina han sido cordiales, pero carentes de sustancia. India se acerca a la región luego de reformas políticas y económicas llevadas a cabo en la década de 1990. Es en la dimensión comercial donde se han concentrado los mayores esfuerzos, aunque aún no se han visto reflejados en aumentos sustantivos en los valores. La región latinoamericana ha utilizado los procesos de integración regional como instrumentos para su desarrollo y como medio para vincularse con otros actores internacionales, como la India. La Alianza del Pacífico, con una vocación hacia el Asia, es un proceso de integración de regionalismo abierto cuyos miembros se encuentran entre los principales socios comerciales de la India en la región. En este sentido, puede cumplir el rol de puente entre la región asiática y América Latina y servir como base para el establecimiento de vinculaciones interregionales. El objetivo del trabajo es realizar un análisis de las vinculaciones de la India con los países miembros de la Alianza del Pacífico. La atención se concentra en los aspectos comerciales y de inversiones, sin dejar de lado lo político, y en las oportunidades y desafíos de dichas vinculaciones.

Palabras clave: India, Alianza del Pacífico, comercio, inversiones, desafíos, oportunidades.

\footnotetext{
* El trabajo se basa en una ponencia presentada en el VIII Congreso Latinoamericano de Ciencia Política, organizado por la Asociación Latinoamericana de Ciencia Política (ALACIP), Lima, julio de 2015

** Doctora en Relaciones Internacionales por la Universidad Nacional de Rosario, Argentina). Profesora e investigadora en la Universidad Siglo 21, en Córdoba, Argentina. Correo electrónico: Paola.Baroni@ues21.edu.ar

*** Analista especializado en relaciones entre India y América Latina. Máster en Estudios Latinoamericanos por la Universidad Stanford, EE.UU. Global Fellow del Wilson Center. Trabajó como investigador en el centro de investigación Gateway House (Mumbai, India) y lideró la sección de América Latina en la Confederación de la Industria India. Correo electrónico: harisesh@alumni.stanford.edu
}

iD https://orcid.org/0000-0002-4146-3429 


\section{India and the Pacific Alliance: opportunities and challenges}

\section{Abstract}

Historically, India's links with Latin America have been cordial, but they lacked of any substance. India approached the region after the political and economic reforms carried out in the 1990s. Even though the commercial dimension was where most efforts have concentrated, there has not been a substantial increase in values. The Latin American region has used regional integration processes as instruments for development and as means to link with other international actors, like India. The Pacific Alliance, with a vocation towards Asia, is an integration process of open regionalism, whose members are the main trading partners of India in the region. In this sense, it can play the role of a bridge between the Asian and Latin American regions and it can serve as a base for building interregional links. The objective of this work is to carry out an analysis of India's links with the member countries of the Pacific Alliance. The focus is on the commercial and investment aspects, without putting aside the political one, and on the opportunities and challenges of those links.

Keywords: India, Pacific Alliance, trade, investments, challenges, opportunities.

\section{La India y América Latina}

Históricamente, las vinculaciones políticas de la India con América Latina y el Caribe (ALC) han sido cordiales, pero carentes de sustancia. La distancia geográfica, la diferencia cultural y política, sumado a distintos procesos históricos, han hecho que tuvieran vinculaciones escasas y esporádicas.

Luego de una profunda crisis económica-financiera a principios de la década de 1990, la India decide liberalizar su economía, lo que le permite su crecimiento económico y favorece su despegue a nivel internacional. Las reformas implicaron la reformulación de la política exterior y comercial, las cuales estuvieron guiadas por el objetivo de crecimiento económico y desarrollo del país. Por eso, el gobierno impulsó el acercamiento hacia regiones y Estados que pudieran contribuir con este objetivo (Tharoor, 2012).

En este sentido, la India ha dividido el mundo en círculos concéntricos para desarrollar su política exterior. El primer círculo lo constituye su vecindario inmediato, Asia del Sur, donde busca su primacía y evitar la injerencia de otras potencias. El segundo círculo es su vecindario extendido (Sudeste de Asia, el Este de Asia, Asia Central, Medio Oriente y el océano Índico), donde ha buscado incrementar su presencia y balancear la influencia de otras potencias, como China. En el último círculo, que incluye el resto del mundo, ha tratado de cumplir un rol de potencia emergente a través de su accionar respecto a la paz y a la seguridad internacional. Aquí se destacan socios estratégicos, como los Estados Unidos y Rusia (Mohan, 2006). Teniendo en 
cuenta esto, se comprende que las vinculaciones con la región latinoamericana no hayan sido más profundas.

Desde su asunción en 2014, el primer ministro Narendra Modi ha viajado a casi todos los continentes. Sin embargo, y a pesar de haber prometido rejuvenecer las relaciones con América Latina, ha realizado solo dos visitas de Estado en marcos multilaterales: la cumbre de los BRICS en Brasil en 2014 y la reunión del G20 en Buenos Aires en 2018. En 2016 realizó una breve visita de trabajo a México (solo cuatro horas) en camino hacia Estados Unidos. Esto indica que, hasta la actualidad, no ha realizado una visita oficial a nivel bilateral al continente. El embajador Deepak Bhojwani (2015) plantea que en la India subsiste una visión simplista y homogénea de Latinoamérica y que en raras ocasiones se comprende una región tan compleja, lo que ha dificultado un correcto acercamiento. El diplomático agrega que si la India busca fortalecer sus relaciones con la región, debe mejorar su valoración de la misma y profundizar su conocimiento respecto a las orientaciones políticas de cada país.

Los flujos de comercio e inversiones entre América Latina y la India siguen siendo bajos, aunque el comercio se ha multiplicado por veinte y las inversiones bilaterales han crecido. Una de las principales críticas es que los gobiernos de ambas regiones no han acompañado el acercamiento con mayor infraestructura y procesos menos burocráticos para lograr una mayor integración (Giordano, Ortiz de Mendívil, Jandhyala y Dutta, 2019). La venta de commodities con destino a la India es la partida que más ha crecido y la que más impacto tiene en la balanza comercial bilateral. Esto se ve reflejado en los casos de Venezuela, México, Colombia y Ecuador con el petróleo; Chile con el cobre; Perú con el oro, y Argentina y Brasil con el aceite comestible. Así, el comercio está concentrado en un escaso número de países y en pocos productos con escaso valor agregado, como son los extractivos y los agrícolas. De esta forma, la región latinoamericana se inserta en la economía india a través de la seguridad alimentaria y energética.

Los Estados latinoamericanos han buscado, históricamente, herramientas y políticas que los potenciaran y proyectaran al continente a nivel internacional. Una de dichas herramientas ha sido los procesos de integración regional. Cada uno con su modalidad, prioridades y crisis, ha buscado vincularse con otros actores internacionales y proyectarse hacia otras regiones, especialmente hacia la asiática en los últimos tiempos (Molano Cruz, 2007). Esta acción encontró, del otro lado del Pacífico, el fuerte interés de aumentar los vínculos con América Latina lo que ha llevado a ambas regiones a replantearse su relación con actores extrarregionales, en un contexto de búsqueda de desarrollo. Habiendo concentrado China la atención, se ha comenzado a dar mayor impulso a otros actores — como la India— que pueden ayudar a 
generar mayor desarrollo a través de la diversificación y a lograr una mejor inserción internacional.

En 2015, los países de Latinoamérica y el Caribe participaron en el Cónclave IndiaALC con el objetivo de explorar opciones para fortalecer el comercio, tanto desde una perspectiva regional como en conjunto. Se presentaron las diversas agrupaciones regionales del continente, como el Mercosur, la Alianza del Pacífico (AP) y el Sistema de la Integración Centroamericana (SICA). El ministro costarricense de Comercio Exterior, Alexander Mora Delgado, indicó que «va a ser más fácil si Latinoamérica se ve a sí misma como un socio comercial de la India, porque entonces ya estamos hablando de dos bloques con unas capacidades de escala que son comparables» (Jabois, 2015). La India, por su parte, se mostró interesada en profundizar los vínculos con los países de la AP.

La Alianza del Pacífico tiene una vocación clara hacia Asia y es un proceso de integración de regionalismo abierto, con un avance en sus objetivos y metas. En este sentido, puede cumplir el rol de puente entre la región asiática y América Latina y servir como base para el establecimiento de vinculaciones interregionales. Además, sus países miembros se encuentran entre los principales socios comerciales de la India en la región. Por ello, el objetivo del trabajo es, en primera instancia, describir las principales características de la política exterior de la India hacia Latinoamérica para luego analizar los vínculos comerciales y políticos con los países de la Alianza del Pacífico: México, Colombia, Perú y Chile. El análisis se desarrolló a través de un diseño de investigación descriptivo y una estrategia metodológica cualitativa. Las técnicas de recolección y análisis de datos utilizadas fueron la observación y análisis de documentos (Valles, 1999).

\section{Características de la política exterior de la India hacia América Latina}

Hasta principios del siglo XXI América Latina permaneció en la periferia de la estrategia económica y política india, ya que no representaba desafíos significativos. Dependiendo del país, ha tenido un contacto limitado y vinculaciones casi inexistentes. La estrategia india de acercamiento hacia la región fue diseñada a finales de la década de 1990 y se sostiene hasta el momento sin mayor innovación.

Un instrumento central - teniendo en cuenta el objetivo de crecimiento económico- ha sido la diplomacia económica, con la que busca promover el comercio exterior y las inversiones (BID, 2010). El principal programa es el FOCUS Lac, creado en 1997 con el objetivo de intensificar las exportaciones indias hacia la región a través del esfuerzo integrado de varias agencias gubernamentales (Kumar, 2010). 
El programa se basa en la asistencia financiera a las empresas indias que quieran participar en ferias comerciales y misiones comerciales a la región y para que realicen estudios de mercado. También se financia el pasaje aéreo y alojamiento local a los empresarios latinoamericanos, para que asistan a eventos comerciales en la India (Viswanathan, 2008). Otra acción es la realización de cónclaves de negocios e inversiones. Los encuentros, organizados por la Confederación de Industria de la India en colaboración con el gobierno indio, son plataformas de contacto entre los diversos actores públicos y privados de ambas regiones.

Aunque ha sido en la dimensión comercial en donde se han concentrado los mayores esfuerzos, aún no se han visto resultados importantes. El papel de la India en el comercio internacional es limitado debido a que cuenta con una apertura comercial más reducida y controlada, y a que tiene elevados niveles de protección (CEPAL, 2012). Las tasas más altas se aplicaron al café, bebidas, bebidas alcohólicas y cereales, productos en los que la región latinoamericana tiene ventaja comparativa. Existen otros tipos de acciones que afectan el comercio bilateral, por ejemplo, la aplicación de cuotas, ajustes de aranceles, aranceles ad valorem, medidas paraarancelarias e impedimentos técnicos. Así, la compleja estructura arancelaria y no arancelaria provoca una elevación de los costos y una mayor incertidumbre para el exportador (Estevadeordal, Mesquita y Kahn, 2017, pp. 21-22). Entonces, la brecha entre el comercio potencial y el real obedece a que el comercio está muy distorsionado, y a que también existen altos costos en las transacciones comerciales y en el transporte (BID, 2010). A esta lista se suma la falta de infraestructura en el país asiático y en algunos países de la región latinoamericana ${ }^{1}$, la falta de conectividad y la ausencia de líneas de transporte marítimo y aéreo directo.

Respecto a los acuerdos comerciales, la India cuenta hasta el momento, con un Acuerdo de Preferencias Fijas con el Mercosur, el cual entró en vigencia en julio de 2009 y tiene un bajo impacto en el comercio, y un Acuerdo Preferencial Parcial con Chile (2007), ampliado en 2016. El otro caso bajo estudio es un acuerdo de libre comercio con Perú. La carencia de acuerdos y lo limitado de los existentes, afecta el despegue del intercambio comercial.

En el periodo 2018-2019, bajó la participación de ALC (3,59\%) en el total del comercio externo de la India debido a la fuerte recesión en varias de las economías latinoamericanas, junto con la caída de los precios internacionales de los productos básicos exportados. También disminuyeron las exportaciones de la India, pasando de un 4,02\% en 2013-2014 a un 2,69\% en 2018-2019. Esta desaceleración se explica

\footnotetext{
1 Se puede mencionar la falta de conectividad al interior de la India y problemas de energía, y en el caso latinoamericano se destaca la falta de conectividad entre la costa atlántica y la pacífica del continente.
} 
por la caída del comercio internacional como así también por el alto nivel de diversificación de proveedores que ha logrado la India (Departamento de Comercio de la India, 2019).

Tabla 1. Principales socios comerciales de India en América Latina y el Caribe (2001-2018) (en miles de millones de dólares)

\begin{tabular}{llcc}
\hline & Principales socios comerciales & Comercio India-ALC & $\begin{array}{c}\text { Participación en el } \\
\text { comercio India-ALC }\end{array}$ \\
\hline 1 & Brasil & 98,76 & $25,09 \%$ \\
2 & Venezuela & 85,65 & $21,76 \%$ \\
3 & México & 59,09 & $15,01 \%$ \\
4 & Chile & 33,73 & $8,57 \%$ \\
5 & Argentina & 27,52 & $6,99 \%$ \\
6 & Colombia & 25,24 & $6,41 \%$ \\
7 & Perú & 16,46 & $4,18 \%$ \\
8 & Ecuador & 6,58 & $1,67 \%$ \\
9 & Panamá & 4,95 & $1,26 \%$ \\
10 & República Dominicana & 4,16 & $1,06 \%$ \\
& Países restantes de ALC & 31,52 & $6,65 \%$ \\
& Comercio total India-ALC & 393,65 & $100 \%$ \\
\hline
\end{tabular}

Fuente: Trade Map, International Trade Center.

Uno de los actores centrales de la inserción comercial internacional de India ha sido su vasto sector externo. Aunque la inversión extranjera directa (IED) en América Latina no es aún significativa, el Departamento de Comercio de la India (2019) indica que han crecido, estimándose en catorce mil millones de dólares (período 2011-2017). Los principales sectores de inversión son los recursos naturales, el farmacéutico, autopartista, minería, hidrocarburos y tecnología de la información y sus respectivos servicios ${ }^{2}$. Mientras las IED chinas en la región se dirigen principalmente a sectores extractivos, las de la India apuntan, además, a las manufacturas y a los servicios, contribuyendo al desarrollo de una mano de obra local calificada y a la transferencia de tecnología (Heine, 2012).

A nivel político-diplomático hubo un esfuerzo por desarrollar las vinculaciones a través del establecimiento de misiones diplomáticas en el continente latinoamericano, pasando de siete representaciones en 2002, a catorce en 2019. Sin embargo, si se

\footnotetext{
2 Entre las principales empresas se encuentran: Tata Consulting Services (IT); Laboratorios Dr. Reddy's y Rambaxy (área farmacéutica); United Phosphorus (agroquímicos); Shree Sugars y Havelis Silvania (equipos de electricidad); Videocon (telecomunicaciones); ONGCVidesh (petróleo), entre otros.
} 
analiza que el continente tiene más de cuarenta países, siguen siendo insuficientes. El gobierno indio también ha desarrollado diversos mecanismos institucionales para revisar los vínculos comerciales y de inversión a nivel bilateral como así también ha creado áreas comerciales y de marketing en las diversas misiones diplomáticas. Además, se observa un incremento en las visitas de Estado (sobre todo de presidentes y vicepresidentes de la India) y en las misiones comerciales (Departamento de Comercio de la India, 2019).

Dentro de este nivel, se destaca el impulso a la cooperación sur-sur, a través de la cual la India busca contribuir al progreso de la región, al mismo tiempo que sirve a diversos intereses nacionales como el de desarrollo. La cooperación se expresa en acuerdos que cubren diferentes temáticas como las tecnologías de la información, la educación, la reducción de la pobreza, la cooperación antártica, entre otras (CEPAL, 2016). No obstante, América Latina recibe menos del 1\% de la Ayuda Oficial al Desarrollo (AOD) que otorga la India a nivel mundial (CEPAL, 2016, p. 77). Por otro lado, no ha utilizado de forma eficiente otros elementos de su poder blando, ya que no ha logrado — salvo en el caso de Brasil— la comercialización de su industria cinematográfica, el desarrollo de interacciones académicas y la eficiencia de programas y becas técnicas y culturales (Bhojwani, 2017).

Entonces, las vinculaciones a nivel político, económico y comercial se encuentran en un estadio inicial con importantes oportunidades para explorar y expandirse. Por lo tanto, la identificación de socios clave con mayor valor para la India es necesaria para así adoptar políticas más eficientes. Dentro de este contexto, la excepción ha sido Brasil, cuyas relaciones se han expandido mucho más que el crecimiento de las vinculaciones con el resto de América Latina (Gangopadhyay, 2014). Por lo expuesto, el trabajo se enfoca en analizar los vínculos y las oportunidades y desafíos que se presentan con otros actores centrales de la región latinoamericana, como son los miembros de la Alianza del Pacífico.

\section{La Alianza del Pacífico y la India}

La Alianza del Pacífico se creó en 2012 y está conformada por Chile, Colombia, Perú y México. Estos países han optado por un regionalismo abierto como vía de desarrollo y crecimiento económico y, por lo tanto, han buscado la construcción de un área de libre circulación de bienes, servicios y capitales. El criterio de membresía estipula que cada país debe tener un Tratado de Libre Comercio (TLC) con cada uno de los miembros restantes. Esto implica que las políticas y regímenes de impuestos son más estables, transparentes y predecibles para la inversión extranjera (Viswanathan, 2015). 
Al no ser un área de integración profunda y contar con una mínima institucionalidad, la AP puede ser caracterizada como de un regionalismo minimalista o un regionalismo económico superficial. Por esto, la AP sigue defendiendo el orden internacional neoliberal vigente y continúa avanzando en la agenda de una inserción económica internacional a través de macro acuerdos (Pastrana Buelvas y Castro Alegría, 2020).

En cuanto a sus principales indicadores económicos, el PBI agregado es de 3800 millones de dólares - que representa un 38\% del PIB de América Latina- y concentra el $50 \%$ de la región con el mundo. Además, cuenta con una población de casi 225 millones de habitantes y una red de más de cincuenta tratados de libre comercio, lo que facilita el acceso a más de sesenta mercados. Se destaca el hecho que los cuatro tienen TLC con los Estados Unidos y con la Unión Europea (Alianza del Pacífico, 2019).

Buscando una posición en el ámbito internacional, la Alianza del Pacífico comenzó una serie de acciones dirigidas a su reconocimiento por parte de otros Estados. Respecto a la India, hizo su presentación como bloque económico ante el empresariado de dicho país en la Cámara de Comercio e Industria en 2012, mostrando las oportunidades de negocios (comercio e inversiones) que el bloque presenta, como así también se expresó la voluntad de estrechar vínculos con el país surasiático en todos los ámbitos (Dinero, 2012). En este sentido, estos países latinoamericanos fueron en búsqueda de inversiones en energías renovables, agricultura, industrias agroalimentarias, minerales y minería (Roche, 2012).

Ante la necesidad de sistematizar y organizar las acciones hacia otros países, la AP creó el Grupo de Relacionamiento Externo (GRE) en 2013. El objetivo es diseñar una estrategia de vinculación con Estados Observadores y terceros Estados con el objetivo de generar un intercambio de forma permanente con los mismos, y así promover los objetivos del bloque y desarrollar los vínculos con estos (Alianza del Pacífico, 2019). En el mismo año, se llevó a cabo en la India la Fine Food India, a la cual la AP fue invitada para ocupar un lugar central. De esta forma, se pone de manifiesto que el bloque es identificado como importante por parte de los organizadores y permitió a los países miembros mostrar en el mercado indio la variedad de productos que pueden ofrecer (Portafolio, 2013).

Con el interés de desarrollar las vinculaciones con el país surasiático, los miembros de la AP realizaron otra presentación, en mayo de 2015, denominando al evento "Alianza del Pacífico: Oportunidades Emergentes», en el Ananta Aspen Centre. A su vez, participaron en el Cónclave India-ALC en Nueva Delhi, presentando el bloque junto a otros procesos de integración regional. Con el impulso dado por estas acciones, organizaron un seminario en Nueva Delhi en octubre de 2016, en el 
que participaron otros países de la región como Argentina, Costa Rica y Guatemala. El objetivo fue compartir experiencias y fortalecer un frente común respecto a un mercado emergente de gran potencial comercial pero que reviste de mucha complejidad, con una importante burocracia y obstáculos lingüísticos y culturales — como la práctica del regateo-, que dificultan los negocios (Emol, 2016).

De esta forma se puede observar que existe un interés por parte de los miembros del bloque en profundizar sus vinculaciones con la India. En este contexto, la India respondió a estas acciones a través de su solicitud de participar como Estado observador, estatus que adquirió en 2014. En tal condición, participa en las Reuniones Ministeriales de Estados Observadores de la Alianza del Pacífico (Departamento de Comercio de la India, 2019), a través de los embajadores indios en el país donde se realiza la reunión. Esta situación dificulta la continuidad de la información y acciones bajo un mismo parámetro, cambiando según sea quien asista a tales reuniones.

Por su lado, las empresas indias han asistido a diferentes macrorruedas de negocios de la AP, en las que participan pequeñas y medianas empresas (pymes) exportadoras de Chile, Colombia, México y Perú (Alianza del Pacífico, 2019). A su vez, han invertido en los países de la AP en áreas como tecnología de la información, productos farmacéuticos, energía, minería y manufacturas. A nivel bilateral también se dan avances en aspectos políticos, culturales y de cooperación científico-tecnológica entre los Estados de la AP y la India, por lo tanto puede utilizarse esa plataforma para avanzar en las vinculaciones.

En 2018, la AP publicó la Visión Estratégica de la Alianza del Pacifico al año 2030, y entre sus puntos centrales se destacan las relaciones externas e interinstitucionales. La idea es sumar más Estados asociados ${ }^{3}$, articular con foros internacionales, profundizar la cooperación económico-comercial con la ASEAN, obtener el estatus de Estado observador de la APEC, entre otras acciones. Esto pone de manifiesto el objetivo del bloque de avanzar en un área de libre comercio con el Asia Pacífico. Además se plantea la intención de promover y avanzar en la cooperación sur-sur con Estados asociados y observadores y con instituciones vinculadas con la AP (Pastrana Buelvas y Castro Alegría, 2020). De lo expuesto se desprende la necesidad de que la AP proyecte un acercamiento más colectivo e institucional hacia la región asiática. En síntesis, estas intenciones y declaraciones de la AP se constituyen en una clara oportunidad para la India de avanzar en la profundización de los vínculos con los Estados del bloque.

\footnotetext{
3 Categoría creada en 2017, hace referencia a cualquier Estado con el cual todas las Partes de la AP celebren y pongan en vigor un acuerdo vinculante de altos estándares en materia económico comercial, que contribuya a la consecución de los objetivos del Acuerdo Marco de la AP (Alianza del Pacífico, 2019).
} 
A continuación, se presenta el análisis de las vinculaciones entre la India y cada uno de los países miembros de la AP.

\subsection{La India y Chile}

Cuando la India declara su independencia en 1947, Chile fue el único país de América Latina presente en la ceremonia y se establecieron las relaciones diplomáticas en 1949. Las consultas políticas se han constituido en el mecanismo de comunicación y canal de coordinación a nivel bilateral y multilateral entre ambos países. Las reuniones se celebran en forma periódica (Embajada de Chile en la India, 2019).

La región del Asia-Pacífico ha sido importante en la política exterior chilena a partir de la década de 1970. El gobierno militar de Pinochet observó que el desarrollo de nuevas relaciones con los países asiáticos podía mejorar la situación de Chile ante las limitaciones derivadas de su situación política internacional de aislamiento, y por eso se observa una activa política exterior hacia esta región, pero es muy limitada respecto a la India. La llegada de la democracia, hacia principios de la década de 1990, no llevó a un cambio respecto a Asia. Los gobiernos continuaron con una política de profundización y fortalecimiento de las relaciones con los países asiáticos debido a la necesidad de reinserción chilena en el mundo. Chile optó por el regionalismo abierto y una política exterior comercial activa, y creó una red de acuerdos comerciales significativa con Asia (Wilhelmy, 2010).

Como en la mayoría de los casos en Latinoamérica, las relaciones tuvieron un desarrollo de baja intensidad hasta la última década del siglo XX. A partir de la década de 2000 se observa un importante despegue de los vínculos, ya que se pueden observar diversas visitas de altos mandos y misiones comerciales (Ministerio de Asuntos Externos de la India, 2018). Por otro lado, y a través de la participación en diversas ferias y de acciones de promoción comercial, el gobierno de Chile ha buscado incrementar los vínculos con la India, mostrando las oportunidades que dicho país ofrece como proveedor de bienes - sobre todo alimentos y bebidas - y servicios y como plataforma confiable para llevar a cabo negocios e inversiones en América Latina (ProChile, 2019).

La India y Chile han trabajado para lograr una vinculación con mayor sustancia, sobre todo en materia económica, comercial y de cooperación técnica. Por ello, se han suscrito acuerdos sobre diversas temáticas, como son: defensa (2007), deportes (2008), cooperación antártica (2008), cooperación en materia sanitaria y fitosanitaria (2001, 2003, 2005), agricultura (2005), educación (2005, 2009), energías renovables (2009), recursos minerales y geología (2009, 2019), servicios aéreos (2008), entre otros (Embajada de Chile en la India, 2019). En 2017, Chile ha firmado el Acuerdo Marco de la Alianza Solar Internacional (Ministerio de Asuntos Externos de la India, 2018). 
A nivel comercial, el principal convenio firmado es el Acuerdo de Alcance Parcial (AAP), que entró en vigencia en 2007, y permitió el despegue del comercio bilateral y la intensificación de las relaciones. En 2014 se amplió el listado de productos pasando de 474 a cerca de 2800. Los productos que se han sumado son aquellos en los que Chile tiene ventaja competitiva, como son, cerezas frescas, cebollas, paltas, uvas, kiwis, mandarinas, duraznos en conserva, entre otros (Ministerio de Asuntos Externos de la India, 2018). En 2019 se acordó avanzar en la ampliación del acuerdo - liberalizar hasta el 90\% de las líneas tarifarias indias-y en diciembre tuvo lugar la primera ronda de negociaciones (Embajada de Chile en la India, 2019; ProChile, 2019). El subsecretario de Relaciones Económicas Internacionales de Chile, Rodrigo Yánez, explicó que el país surasiático es un mercado estratégico para Chile, no solo por la cantidad de población que tiene sino porque es una de las economías más grandes del mundo. Esto, indicó, la presenta como una importante alternativa para las exportaciones de frutas frescas y alimentos en general y para estrechar las inversiones (ProChile, 2019).

Tabla 2. Intercambio comercial India-Chile (2008-2018) (en millones de dólares)

\begin{tabular}{cccc}
\hline Año & Importaciones & Exportaciones & Saldo comercial \\
\hline 2008 & 1342 & 507 & -834 \\
2009 & 1222 & 299 & -923 \\
2010 & 1745 & 417 & -1328 \\
2011 & 1947 & 509 & -1438 \\
2012 & 2541 & 718 & -1822 \\
2013 & 2215 & 738 & -1476 \\
2014 & 2566 & 664 & -1901 \\
2015 & 1884 & 716 & -1167 \\
2016 & 1384 & 727 & -657 \\
2017 & 2072 & 807 & -1265 \\
2018 & 1323 & 976 & -346 \\
\hline
\end{tabular}

Fuente: Trade Map, International Trade Center.

En la tabla 2 se observa la evolución del intercambio comercial y el impacto del AAP en él. Sin embargo, es importante aclarar que en las importaciones desde la India - aunque se observa un crecimiento constante- no hay un salto significativo. La crisis económica y financiera internacional de 2008 provocó un impacto negativo, observándose una caída tanto en las exportaciones como en las importaciones. Un dato que resalta es el constante saldo superavitario para el país sudamericano, convirtiendo a la India en uno de los principales destinos de las exportaciones (ITC, 2020). 
Respecto a la composición del comercio bilateral, hay una alta concentración en un solo producto: el mineral de cobre y sus concentrados, acaparando hasta 2012 un $90 \%$ de lo exportado por Chile a la India. Se identifican, posteriormente, entre 3-4 productos con más del 95\%, sumándose el yodo, la celulosa y el papel. A partir de 2013 comienza una leve diversificación, sumando otros productos derivados del cobre, el concentrado de molibdeno y frutas frescas. En cuanto a las exportaciones indias hacia Chile, se concentran en productos como son los vehículos - autos, camionetas y motocicletas - y maquinaria mecánica con alto valor agregado, constituyendo más del $30 \%$ de lo importado; siguen los medicamentos genéricos, la vestimenta, los textiles, entre otros (ITC, 2020).

El director general de ProChile, Jorge O'Ryan, plantea que las relaciones entre ambos países

[...] tienen enormes oportunidades de complementariedad tanto para frutas contra estacionales como manzanas, kiwis, uvas, ciruelas, cerezas y peras; para alimentos en los que India no es productor neto como la avena, frutos secos como las nueces; para manufacturas de productos químicos y también para hacer uso de herramientas e ideas tecnológicas que nos permitan aunar fuerzas para la innovación y el emprendimiento (ProChile, 2019, s/p).

De acuerdo con datos recabados por el Ministerio de Asuntos Externos de la India (2018), la inversión india en Chile no es significativa y suma aproximadamente unos 216 millones de dólares. Estas se realizan a través de la adquisición de empresas chilenas o del establecimiento de joint ventures. Algunas de las empresas son: Jindal Steel Works, TCS, Oracle, Financial Services, Polaris, Evaluserve, Godrej, Tega Industries, Dr. Reddy's Chile, Seven Pharma, Wipro Technologies, Tata Motors, Bajaj Auto, Mahindra \& Mahindra, Maruti Suzuki, entre otras. En cuanto a las inversiones chilenas en India, estas representan apenas 150 millones de dólares.

Chile es el país único país del bloque que ha logrado firmar y renegociar su acuerdo de preferencias fijas con éxito, logrando no solo la incorporación de más productos, sino que la relación bilateral haya alcanzado una profundidad única.

\subsection{La India y México}

La embajada de México en la India fue inaugurada en 1951, y México fue uno de los primeros en ALC en reconocer su independencia. La persona clave en el acercamiento diplomático entre ambos países en los años posteriores a la independencia, fue Octavio Paz, quien se desempeñó como diplomático y jefe de misión entre 1962 y 1968. Hasta fines de la década de 1980, ambos países profundizaron las relaciones diplomáticas y mantuvieron relaciones activas y cordiales, resultando en siete visitas 
bilaterales de los respectivos jefes de Gobierno (Ministerio de Asuntos Externos de la India, 2020a).

El comercio bilateral ha crecido rápidamente en los últimos ańos, pasando de 262 millones de dólares en 2000-2001 a 9418 millones de dólares en 2018-2019. Esto lo convirtió en el mayor socio comercial de la India en América Latina (ITC, 2020).

Tabla 3. Intercambio comercial India-México (2008-2018) (en millones de dólares)

\begin{tabular}{cccc}
\hline Año & Importaciones & Exportaciones & Saldo comercial \\
\hline 2008 & 1072 & 559 & -513 \\
2009 & 1800 & 684 & -1115 \\
2010 & 983 & 532 & -451 \\
2011 & 983 & 766 & -216 \\
2012 & 2168 & 1337 & -830 \\
2013 & 3495 & 1597 & -1898 \\
2014 & 4295 & 2151 & -2143 \\
2015 & 3445 & 2920 & -524 \\
2016 & 2726 & 2768 & 41 \\
2017 & 2442 & 3375 & 933 \\
2018 & 3532 & 3700 & 167 \\
\hline
\end{tabular}

Fuente: Trade Map, International Trade Center, www.trademap.org

El petróleo crudo desde México y los automóviles desde la India, forman la mayor parte del intercambio comercial: el petróleo crudo representa el 76\% de las exportaciones de México. El petróleo crudo mexicano es tradicionalmente pesado, pero la India cuenta con refinerías complejas que tienen la capacidad de procesar una gran cantidad del mismo. Los automóviles constituyen el $42 \%$ de los envíos de la India a México. En 2018, México representó alrededor del 20\% de las exportaciones totales de automóviles de India, con 1372 millones de dólares. Estas exportaciones son de empresas internacionales instaladas en la India, como Volkswagen, Ford, GM, Chevrolet y Hyundai, y pueden variar dependiendo de las prioridades y planes de mercadeo de cada empresa (ITC, 2020).

Entre otros productos exportados por la India, se encuentran químicos orgánicos, productos farmacéuticos, aluminio, maquinaria industrial y textiles. En cuanto a los exportados por México, se puede mencionar: minerales, productos eléctricos y maquinaria, productos químicos orgánicos, hierro y acero (ITC, 2020). 
Respecto a las inversiones, el Departamento de Comercio de la India (2019) estima que empresas indias han invertido más de tres mil millones de dólares en México. La mayoría de estas inversiones están en tres sectores: automotriz (grupo Samvardhana Motherson, JK Tyres, Spark Minda, RSB Transmissions y el Grupo Varroc); farmacéutica (Dr. Reddy's, Hetero Labs, Lupin, Sun Pharma, Torrent Pharmaceuticals) y tecnología de la información (Tata Consulting Services (TCS), Wipro, Infosys, HCL, Hexaware, Tech Mahindra y NIIT). Además, se incluye empresas de empaque como Uflex, de alimentos como Parle y de agroquímicos como UPL. En cuanto al sector petrolero, la empresa OVL ha firmado un memorando con Pemex en 2014 (Ministerio de Asuntos Externos de India, 2020a).

Por otro lado, según la exembajadora de México en la India, Melba Pria, «México es el mayor inversionista de América Latina en la India, con un total de 800 millones de dólares» (Gandara, 2017, p. 56). Los principales sectores son: autopartes (Nemak, Tremec, Metalsa y Katcon); IT (Softtek); energía; alimentos procesados (Bimbo); productos industriales (Mexichem y Ruhrpumpen) y servicios (KidZania, Cinépolis) (Ministerio de Asuntos Externos de la India, 2020a).

El mayor impulso de las vinculaciones ha venido del sector privado, lo que ha creado un compromiso bilateral más profundo. México se convirtió en el socio comercial más importante de la India en América Latina en 2018, y el flujo comercial entre ambos es mayor que el de India-Rusia o India-Canadá. El futuro de las relaciones India-México es positivo, y los gobiernos de ambos países deben aprovechar esta realidad.

\subsection{La India y el Perú}

India y Perú establecieron relaciones diplomáticas avanzado el siglo XX, en 1963. El Perú abrió su embajada en la India en 1964, y la India recién lo hizo en Lima en 1973. A partir de ese momento han mantenido relaciones cordiales que se han profundizado en el siglo XXI (Ministerio de Asuntos Externos de la India, 2020b).

El comercio bilateral ha crecido recién a partir de 2010, convirtiéndose India en el tercer mayor destino de las exportaciones de Perú en 2018, después de China y EE.UU. En 2001, el comercial bilateral total no superaba los 100000 dólares, pero hacia 2018 alcanzó los 3100 millones. Este acelerado crecimiento se explica por el aumento de las ventas de oro peruano hacia 2012, constituyendo en la actualidad alrededor de dos tercios del comercio bilateral total (ITC, 2020). 
Tabla 4. Intercambio comercial India-Perú (2008-2018) (en millones de dólares)

\begin{tabular}{cccc}
\hline Año & Importaciones & Exportaciones & Saldo comercial \\
\hline 2008 & 297 & 403 & 105 \\
2009 & 69 & 217 & 148 \\
2010 & 211 & 398 & 187 \\
2011 & 429 & 525 & 96 \\
2012 & 420 & 634 & 214 \\
2013 & 692 & 712 & 19 \\
2014 & 563 & 754 & 190 \\
2015 & 746 & 754 & 8 \\
2016 & 989 & 682 & -307 \\
2017 & 2068 & 729 & -1339 \\
2018 & 2472 & 757 & -1714 \\
\hline
\end{tabular}

Fuente: Trade Map, International Trade Center.

El jugador principal en el comercio internacional de oro entre la India y el Perú es MMTC-PAMP, una joint venture entre MMTC, una empresa pública india, y PAMP, un líder a nivel mundial en refinería y lingotes de oro, con sede en Suiza. MMTC-PAMP inició su refinación comercial a gran escala en la India en 2012, cuando el primer cargamento de oro peruano entró en ese país. La empresa tiene una capacidad instalada de refinación de 300 toneladas de oro y compra gran parte del oro peruano exportado a la India (MMTC-PAMP, 2018).

En la composición del comercio se observa una concentración en los minerales, principalmente el oro, con un total de 2400 millones de dólares en 2018. Además, el Perú exporta minerales de cobre y sus concentrados, fosfatos de calcio y minerales de plomo y sus concentrados. También se venden uvas frescas, cacao en grano, palta y harina de pescado. En 2017, el Perú se convirtió en el segundo más grande proveedor de uvas frescas de la India, después de EE.UU., y en uno de los diez principales proveedores de cacao en grano (ITC, 2020).

Las exportaciones de la India al Perú han sido más estables, con un promedio de unos 700000 dólares por año, desde 2012 hasta la fecha. Son mayoritariamente vehículos —automóviles, motos y mototaxis - y autopartes que participan con un casi $20 \%$, luego siguen los textiles, productos farmacéuticos y productos derivados del plástico (ITC, 2020). Dada la complementariedad comercial, ambos países decidieron comenzar los estudios para un acuerdo comercial, que incluya no solo el comercio, sino también elementos de inversión, servicios y relaciones culturales. Hasta marzo 
de 2020 se han llevado a cabo cinco rondas de negociaciones (Ministerios de Asuntos Externos de la India, 2020b).

En el campo de las inversiones, 42 empresas de la India cuentan en la actualidad con oficinas en el Perú, concentradas en sectores de tecnología informática (TI), farmacéutico y automotriz. Una docena de estas inversiones vienen del sector farmacéutico, y la mayoría tienen oficinas de mercadeo o subsidiarios locales para promover sus ventas en el país y en la región andina. Otra media docena de empresas están en el sector automotriz, incluyendo grandes como TVS Motors, Bajaj Auto, Hero Motocorp y Mahindra. La empresa de TI, Tata Consultancy Services (TCS), inició sus operaciones en 2010 y en la actualidad tiene más de mil empleados en el país. Estas inversiones reflejan las tendencias de inversión de la India en América Latina, pero un sector se destaca en Perú: el sector minero. Según el Ministerio de Asuntos Externos de la India (2020b), cinco empresas indias han invertido unos treinta millones de dólares en este sector, incluyendo hierro, carbón y fosforita. La inversión en fosforita es estratégica ya que el fosfato es un elemento indispensable para la elaboración de fertilizantes, de los cuales la India tiene reservas mínimas.

Tabla 5. Empresas indias en Perú, por sector

\begin{tabular}{lcccccc}
\hline Sectores & Farmacéutico & Minería & Tecnología & Autos & Otros & Total \\
\hline No. de empresas & 13 & 11 & 6 & 5 & 7 & 42 \\
\hline
\end{tabular}

Fuente: Entrevistas personales y fuentes de empresas indias, elaboradas por los autores.

Respecto a las inversiones peruanas en la India, se destacan el Grupo AJE, que elabora y comercializa bebidas gaseosas y cuenta con una inversión de más de quince millones de dólares en una planta en Maharshtra; Resemen S.A.C, Opermin y AAC Mining, que venden maquinaria y servicios para el sector minero, y Vistony, fabricante de lubricantes, con una planta en el norte del país (Ministerio de Asuntos Externos de la India, 2020b). La mayoría de estas empresas han invertido en la India con el objetivo de formar parte de las cadenas de valor del país.

Las relaciones bilaterales entre la India y el Perú han sido impulsadas por el comercio internacional y las inversiones, pero necesitan más voluntad política. Los dos países deben concluir, firmar e implementar el Acuerdo Comercial lo antes posible.

\subsection{La India y Colombia}

Aunque las relaciones diplomáticas entre la India y Colombia se establecieron en 1959, recién en 1972 se abrió la embajada colombiana en la India, y en 1973 la de 
India en Colombia. Desde entonces, las relaciones políticas y diplomáticas han sido fortalecidas por el flujo de comercio e inversiones entre los dos países, y el intercambio cultural (Ministerio de Relaciones Exteriores de Colombia, 2018).

El mecanismo institucional establecido entre ambos países es el de las consultas políticas bilaterales, celebrándose reuniones periódicas donde se revisa y trabaja sobre los vínculos en diversas dimensiones. Entre estas consultas políticas, la India y Colombia firmaron dos acuerdos en 2012-2013: el Acuerdo para la Protección y Promoción Recíproca de inversiones (APPRI) y el Acuerdo para evitar la Doble Tributación (ADT).

Aunque las relaciones bilaterales han sido fluidas y cordiales, no se puede decir lo mismo de las visitas de alto nivel entre ambos países ya que los jefes de gobierno de cada país han visitado el otro solo una vez: la primera ministra Indira Gandhi en 1968 y el presidente de Colombia Andrés Pastrana en 2001. Sin embargo, se han llevado a cabo 32 otras visitas de alto nivel, incluyendo ministros y viceministros (Ministerio de Asuntos Externos de la India, 2020c).

Tabla 6. Intercambio comercial India-Colombia (2008-2018) (en miles de millones de dólares)

\begin{tabular}{cccc}
\hline Año & Importaciones & Exportaciones & Saldo comercial \\
\hline 2008 & 22 & 571 & 549 \\
2009 & 336 & 361 & 24 \\
2010 & 765 & 507 & -257 \\
2011 & 713 & 822 & 108 \\
2012 & 1243 & 927 & -315 \\
2013 & 4293 & 1043 & -3249 \\
2014 & 3564 & 1125 & -2439 \\
2015 & 869 & 963 & 93 \\
2016 & 476 & 767 & 290 \\
2017 & 646 & 911 & 265 \\
2018 & 1027 & 1075 & 48 \\
\hline
\end{tabular}

Fuente: Trade Map, International Trade Center.

Los vínculos comerciales y de inversión entre la India y Colombia forman la base más importante de la relación bilateral. El comercio bilateral alcanzó un pico de más de cuatro mil millones de dólares en los años 2013-2014, cuando el petróleo dominaba el comercio bilateral debido a un periodo de precios muy altos a nivel mundial. Adicionalmente, las exportaciones de la India a Colombia han registrado más de mil 
millones de dólares, en promedio, desde 2011. Colombia ha sido el tercer destino más importante para las exportaciones de la India en América Latina desde 2008, después de Brasil y México (ITC, 2020).

Desde 2009, el producto más exportado de India a Colombia ha sido el de motocicletas y, desde 2004, Colombia se ha convertido en uno de los tres principales destinos de exportación de dicho producto a nivel global. El éxito de estas exportaciones se debe a tres razones principales:

a. Tamaño de mercado local. La demanda de motocicletas en Colombia ha aumentado en las últimas décadas — con ventas de unas 600000 unidades al año-, convirtiéndose en el segundo mercado más grande para motocicletas en América del Sur, solo después de Brasil. Esto ha llamado la atención de empresas de India como Bajaj, TVS y Hero (Matthews, 2014).

b. El éxito de Bajaj. El gigante Bajaj, con ventas que superan los 4500 millones de dólares, entró al mercado colombiano en 1993 a través de su socio Auteco, el primer ensamblador de motocicletas de Colombia. Según Auteco, en 2001 Bajaj «inició el ensamble de la Boxer $100 \mathrm{cc}$, la motocicleta más vendida en la historia del país» (Auteco, 2020). Hoy Bajaj tiene la mayor participación en el mercado colombiano de motocicletas, captando aproximadamente un cuarto de las ventas totales (Statista, 2020).

c. La ubicación estrategia de Colombia. La empresa india, Hero MotoCorp, eligió a Colombia como destino para su primera planta internacional de fabricación de motocicletas. Hero es el fabricante más grande de dicho producto en el mundo, e invirtió 70 millones de dólares en una planta en Villa Rica, con una capacidad planeada de producir 150000 unidades al ańo (PTI, 2017).

Además de las exportaciones de motocicletas, India también exporta una importante cantidad de textiles de algodón, químicos orgánicos, productos farmacéuticos, maquinaria, plásticos y otros productos de metalmecánica. Esta canasta de exportación diversificada ha ayudado a India superar los mil millones de dólares en exportaciones desde 2011 (ITC, 2020).

La relación con Colombia se constituye en otro ejemplo de un intercambio interindustrial. El petróleo ha sido el principal producto, seguido por el oro, carbón y otros productos mineros. Sin embargo, las exportaciones de petróleo han sido volátiles debido a los cambios en los precios mundiales de dicho producto. Por ejemplo, las exportaciones fueron de 4200 millones de dólares en 2013, bajando hasta 371 millones de dólares en 2018. Colombia ha exportado una considerable cantidad de productos no-mineros a India, superando los 100 millones de dólares en 2019. Esto incluye productos con valor agregado como la madera teca, plástico en formas 
primarias, productos químicos, maquinaria industrial y otros productos agroalimentarios y de confecciones. Es importante notar que India es el mercado principal para las exportaciones colombianas de madera teca (ITC, 2020).

En cuanto a las inversiones, Colombia es un destino importante para la IED de India. En las últimas dos décadas, las empresas indias han invertido alrededor de mil millones de dólares. La mayor parte de este valor lo representa la empresa estatal de India, ONGC Videsh Limited (OVL), la cual ha invertido más de 650 millones de dólares en campos petroleros colombianos. Esta inversión es parte de una joint venture con la empresa china, Sinopec, llamada Mansarovar Energy, la cual produce más de 30000 barriles de petróleo al día. La empresa tiene 1227 pozos petrolíferos en Colombia y cuenta con 101 millones de barriles de reservas de petróleo (Ministerio de Asuntos Externos de la India, 2020c).

Además, más de 45 empresas indias han invertido en otros sectores de Colombia, como el automotriz, agroquímicos, tecnología informática y farmacéutico. Para todas estas empresas, Colombia es un destino estratégico a largo plazo en Sudamérica, con acceso fácil a todos los mercados de las Américas. Algunas de estas inversiones, como la de Hero y Essel Propack —una empresa especializada en empaques—, se encuentran en las zonas de libre comercio de Colombia (Ministerio de Asuntos Externos de la India, 2020c).

Como se observa, la inversión extranjera de la India en Colombia es de alto valor agregado, centrándose principalmente en manufacturas y servicios y generando un número importante de empleos calificados. Según un estudio del Centro de Estudios sobre la Cuenca del Pacífico, de la Pontificia Universidad Javeriana Cali,

[...] la inversión de la India fue un generador significativo de empleo en Colombia. La entrada de capital de dicha economía, en términos proporcionales, creó más empleo que la inversión procedente de Asia Pacífico y del mundo [...] por cada millón de dólares invertidos en Colombia, la India creó 24,6 empleos, mientras que Asia-Pacífico 3,5 y el mundo 2,7. (Miranda Parrondo, Peláez Soto y Velandia Campos, 2016, p. 99)

Por último, es importante aclarar que entre la India y los Estados miembros de la AP, se ha buscado promover las relaciones culturales. Por ejemplo, Gandhi tiene un significado especial en Colombia, es un símbolo de paz, especialmente durante el conflicto con las FARC; la capital, Bogotá, tiene dos bustos de Gandhi; hay cursos de yoga en las universidades en Colombia, y más de diez mil colombianos participan en eventos de yoga organizados en todo el país (Ministerio de Asuntos Externos de la India, 2020c). 
Colombia es un destino atractivo para la India, tanto por su ubicación estratégica con acceso al Pacífico y Atlántico, como por el tamaño de su población, siendo el segundo país más poblado de Sudamérica. Las empresas colombianas deben mirar más a la India como un destino de inversión para entrar al mercado de Asia, y también como un gran mercado de consumo para todos los productos agroalimentarios, moda y manufactureros.

\section{Reflexiones finales}

En este trabajo se buscó, en primer lugar, caracterizar la política exterior de la India hacia Latinoamérica, para luego analizar los vínculos comerciales y políticos con los países miembros de la Alianza del Pacífico. La primera característica que sobresale es que los vínculos entre la India y los países seleccionados comenzaron a profundizarse recién a partir de comienzos del siglo XXI. Hasta ese momento, los ejes de acercamiento no estuvieron planificados, sino que respondieron más bien a coyunturas internacionales particulares. Las transformaciones del sistema internacional, orientado hacia un mayor multipolarismo, permitieron el acercamiento y aprovechar las ventajas que ofrecía la vinculación de mercados y la concertación política.

Una de las principales observaciones es que la India carece de una política diseñada específicamente para América Latina, teniendo en cuenta las diferentes características económicas, sociales y políticas subregionales. Esta negligencia benigna (Gangopadhyay, 2014; Bhojwani, 2015) se observa no solo por la falta de visitas oficiales bilaterales del primer ministro Modi a los países de la región, sino también por la falta de prioridad dada a bloques regionales que constantemente han mostrado su interés en avanzar en las vinculaciones, como la AP. La India debería aprovechar su estatus de Estado observador y comprometerse con el grupo para avanzar, por lo menos, al estatus de Estado asociado.

Por el lado de la AP, la duda que permanece es sobre la estructura de trabajo que puede ofrecer a los Estados observadores, para incentivarlos no solo a mantener dicho estatus sino a participar activamente. La creación de foros bilaterales, por ejemplo, AP-India sería importante para armar agendas de acuerdo a intereses y necesidades específicas. Otra acción es avanzar en la negociación y concreción de acuerdos y alianzas (públicas y privadas) con la India, con el objetivo de diversificar las exportaciones, las que salvo el caso de México, están concentradas en pocas commodities. La falta de coordinación en las relaciones económicas y comerciales por parte de los Estados de la AP con los países de Asia es evidente, y esto mina la efectividad de los mecanismos del bloque para aprovechar las oportunidades ofrecidas por el dinamismo de la región asiática. 
La dimensión económico-comercial de los vínculos es la que ha contado con mayor cantidad de iniciativas, aunque aún no se han visto resultados importantes. Las elevadas barreras arancelarias y paraarancelarias mantenidas por la India afectan el comercio bilateral, ya que la mayoría de los productos exportados por los países de la AP son recursos naturales y sus manufacturas. Entonces, una acción importante a llevar a cabo es que países como Colombia y México negocien con la India acuerdos comerciales integrales, como lo hizo Chile en su AAP o lo está haciendo el Perú. Esta acción también es importante para la India, ya que permitirá a sus exportaciones competir en mejores condiciones con aquellas de terceros Estados en el mercado de la AP.

Otro elemento importante es avanzar en las inversiones recíprocas. La inversión india no se centra solo en el área extractiva sino que incluye, además, los sectores de la tecnología de la información, el farmacéutico y los vehículos, generando puestos de trabajo calificados. En este sentido, la India debería pensar en otorgar un crédito al bloque para promover y facilitar las inversiones y exportaciones de las compañías indias. Por su parte, los países de la AP deben estimular y ayudar a sus empresarios a participar de las cadenas de valor de la India y a ser jugadores importantes en el gran mercado indio. Además, hay que trabajar en el desarrollo de infraestructura en ambas regiones, sobre todo puertos de gran calado y líneas marítimas y aéreas directas. En este sentido, se hace necesaria la creación de escenarios que faciliten la profundización de las vinculaciones y el aumento de los flujos comerciales e inversiones.

La complementariedad productiva entre la India y los países de la AP indica que existen oportunidades a desarrollar, sobre todo si se toma en cuenta la creciente demanda india de recursos naturales, alimentos y energía, en función del crecimiento de su población y de su desarrollo económico. La concentración de la canasta exportadora de los países de la AP — basada en productos con escaso valor agregadoafecta la forma en que estos se insertan en la economía india: un modelo de inserción basado en la producción primaria y, en consecuencia, más dependiente. Entonces, el desafío para la AP consiste en diversificar su oferta exportable, agregándole mayor valor, y alcanzar esquemas de cooperación tecnológica, científica y energética que permitan continuar y avanzar en la transferencia de tecnología y know-how y así superar el rol primario-exportador. La formación de cadenas de valor dentro de la AP o de forma regional, permitiría una mejor inserción de los países en las cadenas productivas indias y evitar así la competencia con otros actores. El reto está, para ambas partes, en aumentar los estudios de mercado, el conocimiento mutuo más profundo y la difusión de las ventajas mutuas. Si los países robustecen la producción y el mercado interno a través del poder adquisitivo, se aumentarán los contactos y se podrán desarrollar vinculaciones más sólidas. 
Por último, es importante recalcar la necesidad de seguir profundizando las relaciones culturales, para acortar la brecha existente respecto al desconocimiento mutuo. Una acción importante es intensificar las acciones que permitan ver que tanto India como los países de la AP, se presentan como socios naturales y estratégicos, y que pueden ayudarse mutuamente a fortalecer posiciones en los ámbitos multilaterales de negociación.

\section{Referencias bibliográficas}

Alianza del Pacífico quiere hacer negocios con India. (14 de agosto de 2012). Dinero. Recuperado de https://www.dinero.com/negocios/articulo/ alianza-del-pacifico-quiere-hacer-negocios-india/157282

Alianza del Pacífico presenta en la India sus productos. (11 de diciembre de 2013). Portafolio. Recuperado de https://www.portafolio.co/economia/finanzas/ alianza-pacifico-presenta-india-productos-74186

Alianza del Pacífico. (2019). Documentos y estudios. Recuperado de https://alianzapacifico. net/documentos-y-estudios/

Auteco. (2020). Quienes somos. Recuperado de https://www.auteco.com.co/quienes-somos

Banco Interamericano de Desarrollo (BID) (2010). India: oportunidades y desafios para América Latina. Recuperado de http://www.iadb.org/intal/intalcdi/PE/2010/05898. pdf

Bhojwani, D. (2015). Latin America and India: Understanding Mutual Opportunities. Indian Foreign Affairs Journal, 10(1), 50-62.

Bhojwani, D. (2017). Diplomacia Colaborativa. En INTAL Lab-BID (comp.), Latindia. El futuro de la cooperación de India y América Latina (pp. 52-63). Buenos Aires, Argentina: BID-INTAL.

Comisión Económica para América Latina y el Caribe (CEPAL) (2012). La India y América Latina y el Caribe: oportunidades y desafios en sus relaciones comerciales y de inversión. Santiago de Chile: CEPAL-Naciones Unidas.

Comisión Económica para América Latina y el Caribe (CEPAL) (2016). Fortaleciendo la relación entre la India y América Latina y el Caribe. México: CEPAL-Naciones Unidas.

Departamento de Comercio. Ministerio de Comercio e Industria de India. (2019). Annual Report 2018-19. Recuperado de https://commerce.gov.in/writereaddata/uploadedfile/ MOC_637036322182074251_Annual\%20Report\%202018-19\%20English.pdf

Embajada de Chile en la India. (2019). Relación bilateral. Recuperado de https://chile.gob. $\mathrm{cl} /$ india/relacion-bilateral

Estevadeordal, A., Mesquita, M. y Kahn, T. (2017). Hacia una agenda común de comercio. En INTAL Lab-BID (comp.), Latindia. El futuro de la cooperación de India y América Latina (pp. 14-33). Buenos Aires: BID-INTAL.

Gangopadhyay, A. (2014). India-China Competitions in Latin America: Some Observations. Journal Global \& Strategis, 8(1), 1-13. 
Gándara, G. (2017). México e India: futuro cercano. Entrevista con Melba Pría, Embajadora de México en India. Comercio Exterior. Bancomext, (9), enero-marzo, 54-57. Recuperado de https://www.revistacomercioexterior.com/revistas/9/1494000903494.pdf

Giordano, P., Ortiz de Mendívil, C., Jandhyala, V. y Dutta, A. (2019). Un puente entre América Latina y la India. Políticas para profundizar la cooperación económica. Washington DC: BID-Eximbank. http://dx.doi.org/10.18235/0001733

Heine, J. (2012). The New India and the New Latin America. Hemishphere, 21, 31-33.

International Trade Center (2020). Trade Map. Recuperado de www.trademap.org

Jabois, N. (8 de octubre de 2015). Latinoamérica trata de conquistar la India regionalmente y en bloque. EFE. Recuperado de https://www.efe.com/efe/america/economia/latinoamerica-trata-de-conquistar-la-india-regionalmente-y-en-bloque/20000011-2733553

Kumar, R. (2010). India expanding relations with Latin America and Caribbean. The extraordinary and Plenipotentiary Diplomatist-Plus, May, 10-13.

Matthews, H. (7 de Julio de 2014). Leading global motorcycle producer invests $\$ 70 \mathrm{M}$ for new factory in Colombia. Colombia Reports. Recuperado de https://colombiareports. com/leading-global-motorcycle-producer-chooses-colombia-new-base/

Ministerio de Relaciones Exteriores de Colombia. (Enero de 2018). Informe de Gestión 2017. Recuperado de https:/www.cancilleria.gov.co/sites/default/files/FOTOS2018/ informe_de_gestion_institucional_2017_1.pdf

Ministerio de Asuntos Externos de la India (2018). India-Chile Bilateral Relations. Recuperado de http://mea.gov.in/Portal/ForeignRelation/India_Chile_Bilateral_Brief_5_18. pdf

Ministerio de Asuntos Externos de la India (2020a). Mexico Bilateral Brief. Recuperado de http://mea.gov.in/Portal/ForeignRelation/Mexico_Bilateral_Brief_Jan_2020.pdf

Ministerio de Asuntos Externos de la India (2020b). India-Peru Relations. Recuperado de http://mea.gov.in/Portal/ForeignRelation/Peru_Bilateral_brief_January_2020.pdf

Ministerio de Asuntos Externos de la India (2020c). Brief on Colombia Bilateral Relations. Recuperado de http://mea.gov.in/Portal/ForeignRelation/Colombia_bilateral_Brief_ Jan_2020.pdf

Miranda Parrondo, M., Peláez Soto, J. T. y Velandia Campos, S. (2016). Colombia y la India: Relaciones de Comercio, Inversión y Oportunidades de Intercambio. Journal of Business, 8 (1), 91-109. https://doi.org/10.21678/jb.2016.78

MMTC-PAMP. (2018). India Pvt. Ltd. Recuperado de https://www.mmtcpamp.com/

Mohan, R. C. (2006). India and the Balance of Power. Foreign Affairs, 85(4), 17-32. https:// doi.org/10.2307/20032038

Molano Cruz, G. (2007). El interregionalismo y sus límites. Estudios Internacionales, (158), 9-27.

Países de Alianza del Pacífico promueven frente comercial común en la India. (18 de octubre de 2016). Emol. Recuperado de https://www.emol.com/noticias/ Economia/2016/10/18/826965/Paises-de-Alianza-del-Pacifico-promueven-frentecomercial-comun-en-la-India.html 
Pastrana Buelvas, E. y Castro Alegría R. (2020). Auge y estancamiento de la Alianza del Pacífico. Análisis Carolina, 7. https://doi.org/10.33960/AC_7.2020

ProChile. (26 de agosto de 2019). Chile e India inician negociación para ampliar Acuerdo Comercial. Recuperado de https://www.prochile.gob.cl/noticia/ chile-e-india-inician-negociacion-para-ampliar-acuerdo-comercial/

PTI.(27 dediciembrede2017). HeroMotoCorpbeginsoperationsatColombiaplant; firstoutside India. Financial Express. Recuperado de https://www.financialexpress.com/industry/ hero-motocorp-begins-operations-at-colombia-plant-first-outside-india/132877/

Roche, E. (14 de agosto de 2012). Pacific Alliance seeks investment from India. LiveMint. https:/www.livemint.com/Politics/h1rIV10gcAPGZ77UM6fmeK/Pacific-Allianceseeks-investment-from-India.html

Statista (2020). Estadísticas para datos de mercados. Recuperado de https://es.statista.com/

Tharoor, Sh. (2012). Pax Indica. India and the World of the Twenty-firt Century. Nueva Delhi: Pinguin Books.

Valles, M. (1999). Técnicas cualitativas de investigación social: Reflexión metodológica y práctica profesional. Madrid: Síntesis S.A.

Viswanathan, R. (2008). Socios a largo plazo. Res Diplomática. Segunda época, (3), 68-77.

Viswanathan, R. (2015). The Pacific Alliance \& India. Gateway House. Recuperado de https://www.gatewayhouse.in/the-pacific-alliance-india/

Wilhelmy, M. (2010). La trayectoria de Chile frente a la región Asia-Pacífico. Estudios Internacionales, 43(167), 125-141. https://doi.org/10.5354/0719-3769.2010.12701

Recibido: 6 de abril de 2020

Aprobado: 30 de junio de 2020 